\title{
COMMUNICATION
}

\section{Extractions dentaires et troubles de l'hémostase : étude cas-témoin au CHU de Rennes}

\section{Dr Bourhy $\mathbf{0}^{1}$, Dr Guillet B², Dr Limbour $\mathrm{P}^{3}$}

1 - dentiste spécialiste en chirurgie orale

2 - médecin spécialiste en biologie médicale, MCU-Ph au CHU de Rennes

3 - dentiste spécialiste en chirurgie orale, MCU-Ph au CHU de Rennes

\section{Introduction}

Cette communication rapporte une étude rétrospective monocentrique cas-témoin comparant le risque hémorragique après extraction dentaire entre les patients atteints de Troubles Hémorragiques Constitutionnels ( $\mathrm{THC}$ ), les patients sous traitement anti thrombotique (antiagrégants plaquettaires : acide acétylsalicylique, clopidogrel ; anticoagulants : AVK) et les patients sains (aucun trouble hémorragique constitutionnel ou acquis). À notre connaissance, aucune autre étude établissant cette comparaison n'a été publiée dans la littérature internationale.

Le but de cette étude est de comparer les différents protocoles d'hémostase suivis dans notre centre lors d'avulsions dentaires chez des patients avec différents risques hémorragiques.

Cette étude a reçu l'avis favorable du comité d'éthique du CHU de Rennes (avis n¹4-85).

Patients et méthodes

4 groupes de patients ayant eu des extractions dentaires dans notre centre ont été consécutivement inclus : patients avec THC à l'exclusion de toute autre anomalie acquise de I'hémostase (119 patients pour 127 interventions entre 2006 et 2015), patients sous AVK (28 patients, 28 interventions - 2013-2014), patients sous AAP (21 patients, 21 interventions - 20132014) et un groupe contrôle (35 patients, 35 interventions - 2013-2014).

\section{Résultats}

628 dents ont été extraites au total (THC : 390 dents, groupe AVK : 74 dents, groupe AAP: 69 dents, groupe contrôle : 95 dents). Aucune complication grave n'est survenue sur l'ensemble de l'étude. 23 patients ont eu un épisode de saignement post-opératoire (THC : 9, AVK : 7, AAP : 3, contrôle : 4), parmi lesquels 6 ont nécessité une aide médicale (THC : 3, AVK : 3). L'incidence des saignements était significativement plus élevée dans le groupe AVK.

\section{Discussion}

Nos résultats sont équivalents à ceux obtenus par d'autres études. En comparaison aux patients traités par AVK, une simplification peuvent être envisagées dans la prise en charge des patients THC, à part pour les troubles sévères.

\section{Conclusion}

Cette étude permet d'établir un premier lien dans la prise en charge des patients avec différents risques hémorragiques lors d'avulsions dentaires. 